\title{
BROTE INTRAHOSPITALARIO DE SALMONELOSIS POR SALMONELLA ENTERITIDIS SEROTIPO AGONA
}

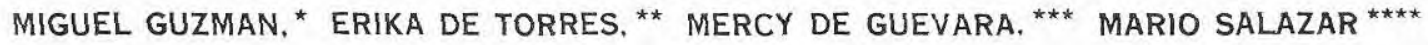

\begin{abstract}
Se describe una epidemia de salmonelosis ocurrida en el servicio de Pediatría del Hospital Militar, la cual afectó a quince pacientes. Se concluye que fue una infección intrahospitalaria. Como agente etiológico fue plenamente identificado Salmonella enteritidis, serotipo agona con una gran multirresistencia a los antibióticos. Como orígen posible del brote se demostró un portador sano, preparador de teteros en el servicio. Es, posiblemente, la primera vez que se informa Salmonella agona en nuestro país.
\end{abstract}

\section{INTRODUCCION}

Los brotes epidémicos intrahospitalarios son hechos de común ocurrencia; casi siempre son debidos a microorganismos del medio hospitalario (1), frecuentemente de tipo oportunista (2); pero también, pueden ocurrir brotes causados por patógenos primarios como los del género Salmonella $(3,4,5)$. El presente estudio describe un brote de salmonelosis ocurrido en un hospital general que por su forma de presentación, agente etiológico y comportamiento frente a los antibióticos, es particularmente interesante.

\section{MATERIALES Y METODOS}

\section{Cepas:}

Los microorganismos del estudio fueron cepas aisladas de casos activos de gastroenteritis referidos a la Unidad de
Enterobacterias del Instituto Nacional de Salud.

Medios de Cultivo:

Para el estudio e identificación de los microorganismos se siguió el esquema estandarizado en la Unidad de Enterobacterias según recomendaciones de los centros para control de enfermedades de los Estados Unidos (CDC - Atlanta) (6).

\section{Sueros Tipificadores:}

Para la identificación final de los microorganismos se utilizaron antisueros monoespecíficos, comercialmente obtenidos de la casa alemana Berhing.

\section{Antibiograma:}

Para las pruebas de sensibilidad a los antibióticos se utilizaron el medio Mueller-

\footnotetext{
* Jefe Sección de Diagnóstico, Investigación y Referencia, Instituto Nacional de Salud, Bogotá. Profesor Asociado, Facultad de Medicina, Universidad Nacional, Bogotá.

** Jefe Servicio de Pediatría, Hospital Militar Central, Bogotá.

*** Bacterióloga Unidad de Enterobacterias, Grupo de Microbiología e Inmunología, Instituto Nacional de Salud, Bogotá.

**** Epidemiólogo, Crupo de Microbiología e Inmunología, Instituto Nacional de Salud, Bogotá.
}

Investigación realizada con el apoyo del Programa de Cooperación de la Agencia Internacional de Cooperación del Japón y el Instituto Nacional de Salud de Colombia. 
Hinton (BBL), discos comerciales (Difco) y la técnica de Kirby-Bauer, según normalización internacional (7).

\section{Descripción del Brote:}

El 17 de junio de 1981 se aisló en el laboratorio de Bacteriología del Hospital Militar un microorganismo identificado presuntivamente como Salmonella sp. Este microorganismo provenía de un coprocultivo de un paciente del servicio de pediatría. El paciente había sido dado de alta seis días antes, por mejoría, luego de ser tratado para un proceso de enfermedad diarréica aguda (EDA).Referida la cepa al INS se identificó como Salmonella enteritidis, serotipo agona. El mismo día 17, se aisló en el hospital una Salmonella sp. de un catéter pleural retirado de un niño que llevaba once días de hospitalización con diagnóstico de neumonía y paquipleuritis; el 19 de julio se aisló Salmonella sp. de un coprocultivo de este mismo paciente que en ese momento presentaba además un cuadro diarreico; ese mismo día se aisló Salmonella sp. en el coprocultivo procedente de otro niño hospitalizado el día anterior con diagnóstico de sarampión. Las tres cepas fueron estudiadas en el INS y finalmente identificadas como Salmonella enteritidis, serotipo agona.

Simultáneamente se aisló Salmonella sp. de un cuarto niño con diarrea que correspondió también a Salmonella enteritidis, serotipo agona. El 18 de julio se aisló una salmonella sp. en el coprocultivo de un niño nacido el 9 de julio en la institución que debió permanecer hospitalizado por cuadro neurológico y pulmonía neonatal con diarrea sobreagregada a partir del 17 de julio. En las semanas subsiguientes se aislaron en el laboratorio del hospital cepas de Salmonella sp. procedentes de coprocultivos de otros ocho pacientes del servicio de pediatría; uno de estos pacientes permanecía hospitalizado por cardiopatía congénita y otro, tratado por un traumatismo craneano, dado de alta cinco días antes, y reingresado después por cuadro diarréico. Cuatro de los pacientes ingresaron al servicio por diarrea, y luego de permanecer en el servicio presentaron mejoría clínica pero recayeron con diarrea grave con deposiciones frecuentes, acuosas, fétidas, de color verdoso. Estudiadas las cepas se identificaron todas como Salmonella enteritidis, serotipo agona. Ante la sospecha clara de un brote epidémico se tomaron las medidas conducentes a aclararlo e identificar su causa mediante coprocultivos del personal médico y paramédico de la unidad. Del coprocultivo de un preparador de teteros se aisló e identificó una Salmonella enteritidis serotipo agona, retirado del servicio, cesó la ocurrencia de casos. Seis semanas después del último caso, el 17 de septiembrs, se aisló Salmonella enteritidis, serotipo agona, del coprocultivo de un niño nacido en la Institución seis días antes y que desarrolló un cuadro diarreico durante la observación perinatal; a los tres días se aisló Salmonella enteritidis, serotipo agona del coprocultivo de un niño convaleciente de un cuadro diarréico que recayó con una sintomatología caracterizada por deposiciones acuosas, fétidas y verdosas.

Al estudiar nuevamente la situación se encontró que el preparador de teteros había sido reintegrado al servicio luego de haber sido tratado con cloranfenicol. Un nuevo coprocultivo de este empleado demostró una vez más Salmonella enteritidis, serotipo agona. El empleado fue retirado definitivamente, lo que trajo el cese de casos por dicho organismo.

En resumen, se estudiaron quince casos intrahospitalarios, de cada uno de los cuales se aisló e identificó Salmonella enteritidis, serotipo agona, distribuidos así: Cuatro en la primera semana de observación, nueve en la semana sexta y octava y dos en la semana catorce; además de éllo, se tiene un preparador de teteros, portador sano del microorganismo, del cual fue aislado en la semana octava y catorce (Fig, 1). Las 15 cepas procedentes de coprocultivos, una de sangre y una de un catéter fueron cuidadosamente confirmadas en el INS como Salmonella enteritidis, serotirpo agona, y enviadas al laboratorio de Enterobacterias del CDC en Atlanta, en donde fue confirmada dicha identificación. 


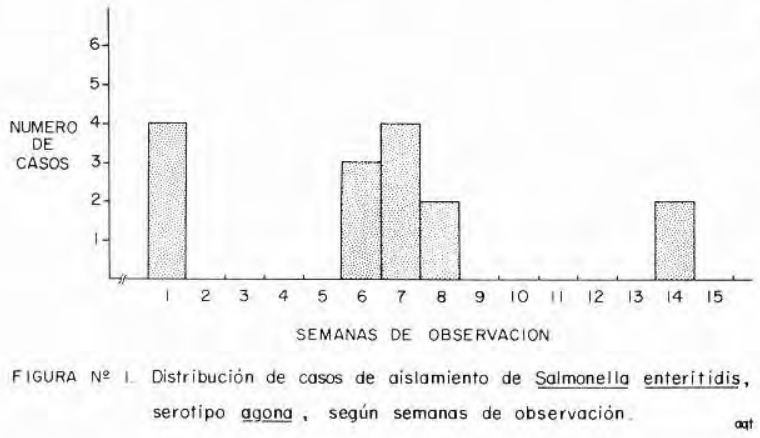

CUADRO N2 I Sensibilidad de las cepas de Salmonella enteritidis, serotipo agona aisladas Antibiograma de discos.

\begin{tabular}{|l|c|c|c|c|c|c|c|}
\hline \multirow{2}{*}{ ANTIBIOTICOS } & \multicolumn{2}{|c|}{ RESISTENTES } & \multicolumn{3}{|c|}{ SENSIBLES } & \\
\cline { 2 - 6 } & $\begin{array}{r}10 \\
\mathrm{~mm}\end{array}$ & $\begin{array}{c}11-15 \\
\mathrm{~mm}\end{array}$ & $\begin{array}{c}16-20 \\
\mathrm{~mm}\end{array}$ & $\begin{array}{c}21-25 \\
\mathrm{~mm}\end{array}$ & $\begin{array}{c}26-30 \\
\mathrm{~mm}\end{array}$ & $\begin{array}{r}31 \\
\mathrm{~mm}\end{array}$ & TOTAL \\
\hline AMIKACINA & & & & 14 & 1 & & 15 \\
AMPICILINA & 15 & & & & & & 15 \\
CEFAMANDOLE & & 13 & 2 & & & & 15 \\
CEFOTAXIMA & & & & & 6 & 9 & 15 \\
CEFOXIIINA & & & & 15 & & & 15 \\
CEFALOTINA & 12 & 3 & & & & & 15 \\
CLORANFENICOL & 15 & & & & & & 15 \\
GENTAMICINA & 14 & 1 & & & & & 15 \\
KANAMICINA & 15 & & & & & & 15 \\
SULFA/TRIMETROPIN & & 1 & 14 & & & & 15 \\
TETRACICLINA & 15 & & & & & & 15 \\
TOBRAMICINA & 14 & 1 & & & & \\
\hline
\end{tabular}

Cada una de las cepas fue estudiada para conocer su sensibilidad a los antimicrobianos mediante la técnica de Kirby-Bauer (7).

El patrón de sensibilidad fue idéntico para amikacina, cefotaxima, cefoxitina y trimetropin-sulfa (cuadro No. 1).

\section{DISCUSION}

Salmonella enteritidis, serotipo agona es un micro-organismo de aislamiento frecuente en productos de orígen veterinario reconocido como agente causal de gastroenteritis en varias regiones geográficas, tanto en adultos como en niños; ocupa en los Estados Unidos un lugar importante, 5o por su frecuncia (8) y en algunos países latinoamericanos como Chile, México y Venezuela (9); en nuestra patología colombiana no tenemos información al respecto siendo posiblemente esta la primera vez que se informa como agente causal plenamente identificado de un brote epidémico de salmonelosis. El análisis global del estudio nos pemite concluir que se trató de un brote epidémico con tres períodos de diseminación intrahospitalaria, ya que ocurrieron varios casos en lapsos cortos de una a tres semanas. No hubo aparición de nuevos casos durante un período de observación considerable a partir de la $14^{\mathrm{B}}$. semana. La Salmonella agona apareció en un niño que para el brote puede considerarse como el caso índice: el estudiado el 17 de julio y tratado para un proceso respiratorio. Como orígen de la epidemia posiblemente puede señalarse al preparador de teteros, portador del microorganismo. El brote debe considerarse como infección intrahospitalaria por las siguientes consideraciones:

1. Aparición de cuadros diarreicos o modificaciones de los existentes en niños hospitalizados.

2. Aislamiento de un mismo microorganismo el día del evento.

3. Ocurrencia en tres niños, los cuales habiendo nacido en la institución, permanecían en el servicio por diversas razones clínicas.

4. Reaparición del problema cuando se reincorporó al servicio el portador.

\section{Finalización al retiro definitivo del portador.}

Es interesante destacar, una vez más, la necesidad de estudiar cuidadosamente las cepas de Salmonella circulantes en nuestro medio con el objeto de tener una mayor precisión del manejo clínico. En el caso presente si no hubiese tenido el hospital Militar el interés de referir las cepas el episodio epidémico habría pasado inadvertido o se habría prolongado y extendido.

La salmonelosis es una infección en extremo frecuente en múltiples lugares (10-17); aunque Salmonella enteritidis, serotipo agona tiene una identificación química que permite identificarla como género, su fórmula antigénica: antígeno somático $\mathrm{O} 4,12$ y flagelar fgs requiere una 
completa provisión de antisueros para poder llegar a su tipificación final (tabla No.1) lo cual, escapa a la mayoría de los laboratorios de las instituciones de atención médica, razón por la cual, no se informa nunca; es por éllo que desconocemos casi por completo cuales especies y serotipos circulan en nuestra población excepto Salmonella typhi (16). Parece por este estudio y por observaciones posteriores que S.agona es un importante patógeno como ocurre en otros países (18). La cepa aislada como agente causal de la epidemia descrita tiene el interés adicional de una gran resistencia a los antibióticos, 8 de los 15 utilizados; los estudios preliminares que hemos realizado indican que esta resistencia múltiple está posiblemente mediada por un plásmido, lo cual demuestra el peligro de diseminación de éste en un medio hospitalario y en la comunidad como ya ha sido establecido en

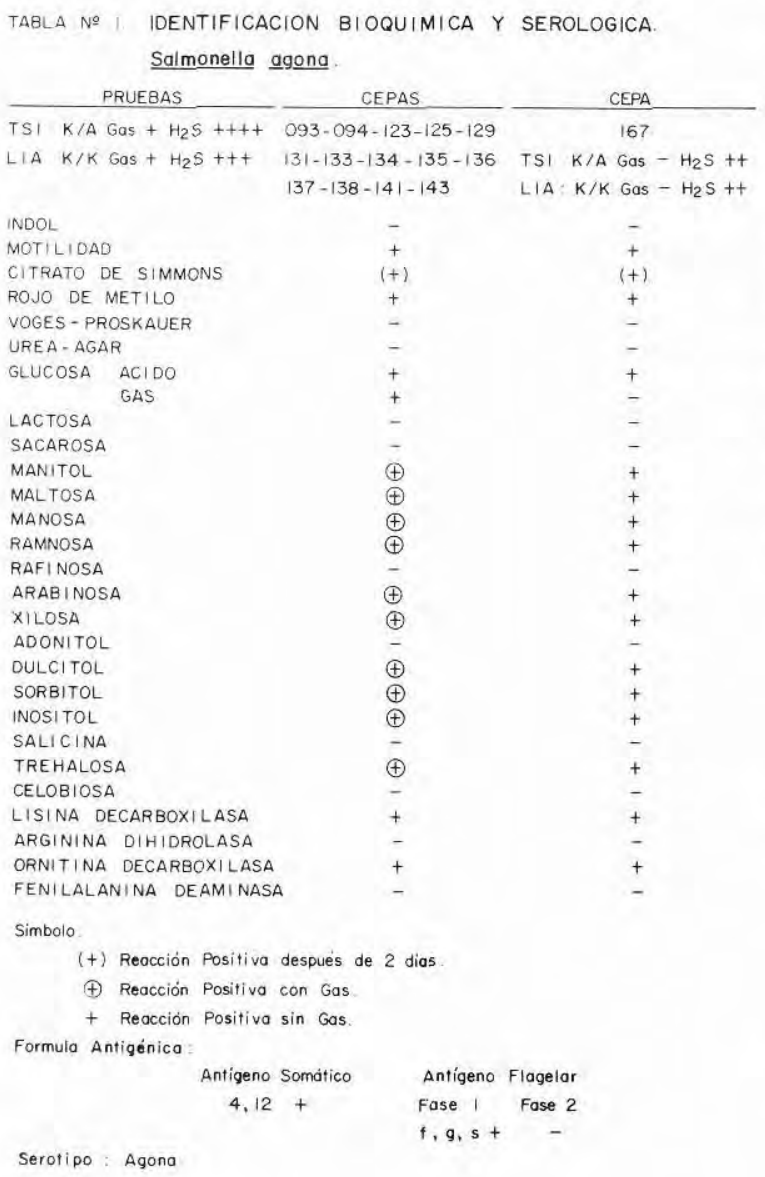

otros casos similares (19). Este hallazgo de multiresistencia es coincidente con lo descrito por otros autores para muchos otros serotipos de salmonellas (20-23).

\section{SUMMARY}

An epidemic outbreak of salmonellosis detected in the pediatric unit of the Central Military Hospital (Bogotá Colombia) with fifteen instances is presented hereby. The study showed that the outbreak was an intrahospitalary infection. Salmonella enteritidis, serotype agona was identified as the aetiological agent; the microorganism exhibited a high degree of multiresistance to antimicrobials.

A healthy carrier who prepared the nursing bottles was found as the most posible originator of the outbreak. This is the first time that Salmonella agona is reported in our country.

\section{AGRADECIMIENTOS}

Queremos expresar nuestro agradecimiento al personal del Laboratorio de Bacteriología del Hospital Militar, por su eficiente colaboración e interés, al doctor Alvaro Aguilera B., por el análisis de los datos y al doctor James $R$. Allen, Jefe de la Sección de Investigaciones Epidemiológicas, del Programa de Infecciones Hospitalarias de los Centros para Control de Enfermedades (CDC) de Atlanta.

\section{BIBLIOGRAFIA}

1. John J F, Sharbaugh R J. Bannister E R. Enterobacter cloacae: Bacteremia, epidemiology and antibiotic resistance. Rev. Inf. Dis. 1982; 4: 13.

2. Gardner P, Griffin WB, Swartz M N, Kunz L J. Nonfermentative gram-negative bacilli of nosocomial interest. Am. J. Med. 1970; 48: 735.

3. Mackerras IM, Mackerras M J. An epidemic of infatile gastro- nteritis in Queensland caused by Salmonella bovis morbificans (Basenau). J. Hyg. 1947; 49:166. 
4. Sohroeder SA, Aserkoff B, Brachman PS. Epidemic Salmonellosis in Hospitals and Institutions. A Five year Review. New Eng. J. Med. 1968; 279: 674 .

5. Hirsch W, Konigsberg MD, Sapiro-Hirsh R, et al. Salmonella edinburg infection in children. A protracted Hospital Epidemic Due to a MultipleDrug resistance Strain Lancet. 1965; 2: 828.

6. Ewing WH, Davis BR. Media and tests for Differentiation of Enterobacteriaceae. U.S. Dept. Health, Ed. Welfare. 1975; Publication No. (CDC) 76-8236.

7. Thornsberry C, Hawkins T H. Agar Disc Diffusion Susceptibility Testing Procedure, U.S. Dept. Health, Ed, Welfare. Public Health Service. Center For Disease Control.

8. Center For Disease Control Salmonella Surveillance, U.S. Department of Health and Human Services Public Health Service Annual Summary 1979. Issued Nov. 1981.

9. Comunicación personal. Curso de Vigilancia de Resistencia a los Antibióticos OPS. Caracas octubre 1984.

10. Centers for Disease Control. Salmonella dublin Associated with Raw Milk - Washinton State MMWR. 1981; 30: 373 .

11. Gunn RA, Markakis G. Salmonellosis associated with homemade Ice Cream. An outbreak report and Summary of outbreaks in the United States in 1966 - 1976 JAMA 1978; 240: 1885.

12. Fontaine RE, Cohen ML, Martin WT et al. Epidemic Salmonellosis from cheddar cheese: Surveillance and prevention. Am. J. Epidemiol. $1980 ; 111: 247$.

13. Graven PC, Mackel DC, Baine W B et al. International outbreak of Salmonella eastbourne infection traced to contaminated chocolate. Lancet 1975 ; i: 788 .

14. Werner SB, Humphrey GL, Kamei I. Association Between raw milk and human Salmonella dublin infection Brit. Med, J. 1979; 2: 238.

15. Center for Disease Control. Salmonellosis from homemade ice-Cream Georgia MMWR 1981; 30: 467 .

16. Prada G, Guzmán M. Fiebre Tifoidea. Estudio de tres brotes Epidémicos Act. Med. Col. 1982; 7: 81.

17. Center for Disease Control. Multiple outbreaks of Salmonellosis associated with precooked roast beef Pennsylvania, New York, Vermont. MMWR 1981; 30: 570 .

18. Center for Disease Control. Human Salmonella isolates United States, 1980 MMWR 1981; 30: 377.

19. O Brien TF, Hopkins JD, Gilleece ES, et al. Molecular epidemiology of antibiotic resistance in Salmonella From animals and human beings in the United, States. New Eng J. Med. 1982; 307: 1.

20. Centers for Disease Control. Multiresistant Salmonella and other infections in adopted infants from India M M W R. 1982; 31: 285.

21. Falbo V, Capridi A, Mondello F. et al. Antimicrobial resistance among Salmonella isolates from hospitals in Rome J. Hyg, Camb. 1982; 88: 275.

22. Ryder RW, Blake PA, Murlin AC. et al. Increase in antibiotic resistance among isolates of Salmonella in the United States, 1967-1975 J. Inf. Dis. $1980 ; 142$ : 485.

23. Rowe B, Frost J A, Threlfall EJ. et al. Spread of a multiresistant clone of Salmonella typhimurium phage type $66 / 122$ in South-East Asia and the Middle East. Lance, 1980; 1: 1070. 